\title{
Enhancing the Life Cycle Sustainability of Camelina Oil-Based Acrylic Monomer
}

\author{
Iryna Bon ${ }^{1}$, Na Wu², Zoriana Demchuk ${ }^{3}$, Andriy Voronov', Ghasideh Pourhashem ${ }^{1}$ \\ ${ }^{1}$ Department of Coatings and Polymeric Materials, North Dakota State University, Fargo, ND \\ Iryna.Bon@ndsu.edu; Andriy.Voronov@ndsu.edu; Ghasideh.Pourhashem@ndsu.edu \\ ${ }^{2}$ Yale school of the environment, Yale university, New Haven, CT \\ na.wu@yale.edu \\ ${ }^{3}$ Chemical Sciences Division, Oak Ridge National Laboratory, Oak Ridge, TN \\ demchukz@ornl.gov
}

\section{Extended Abstract}

Using plant oils for the synthesis of biobased monomers can potentially provide an opportunity for improving the environmental performance of the polymeric materials. One-step method that converts plant oils into acrylic monomers for free radical polymerization, including emulsion processes to yield latex polymers has been developed in our group. Synthesis of monomers from various oils differs in terms of yield, cost and environmental performance based on plant sources.

The major challenge of utilization plant oils in chemical industry is finding the feedstock with consistent composition and non-edible sources which do not compete with food production. Camelina sativa has several agronomic advantages in comparison to traditional commodity oilseed crops (canola, sunflower, soybean, etc.), including low requirement of water and fertilizer during cultivation, adaptability to various environmental conditions and resistance to pathogens. Additionally, Camelina is used as feedstock for animal meal, so it does not compete with food productions. Moreover, camelina seed oil having high amount of unsaturated double bonds in the fatty acids is potential candidate for new biobased monomer production and further utilization in adhesive production.

In this study, life cycle assessment (LCA) methods with cradle-to-gate approach were used as a guide, early in the design of the acrylic monomer from camelina oil, to evaluate its environmental performance and optimize the synthetic pathway. Initially, preliminary LCA model of the camelina oil-based monomer (CMM) synthesis was built based on the experimental data and literature. Using SimaPro software, influence of each component used in the monomer synthesis was investigated within 10 main impact categories such as ozone depletion, global warming potential, acidification, eutrophication carcinogenic and noncarcinogenic human health hazards, ecotoxicity, respiratory effects, smog formation, and fossil fuel depletion to identify the hotspots in the synthesis.

LCA results showed that camelina oil extracted from the camelina seed was the major contributor to the eutrophication category due to use of fertilizer and pesticide. However, oil is produced from the biomass that consequently provide carbon credit to the system, lowering the impact in the global warming category.

Results show that used as a solvent during the synthesis tetrahydrofuran (THF) and electricity consumed during all preparation steps are the main contributors to fossil fuel depletion impact category due to non-optimized lab-scale process. Additionally, using dichloromethane (DCM) - solvent with low boiling point - for monomer purification after synthesis results in high losses to atmosphere and can cause serious environmental and human health risks. Thus, synthesis of CMM was performed at the lab-scale varying two alternative solvents with better environmental performance in comparison to DCM - hexane and 2-methyl-tetrahydrofuran (Me-THF). 6\% higher yield and significantly lower environmental impact in all categories (except of ozone depletion where the impact diminishes dramatically in comparison to dichloromethane) was observed.

Laboratory data from the modified CMM synthesis with cosolvents Hexane and Me-THF was used for further modelling of the larger scale production using process simulation with Aspen Plus software. Energy optimization and both solvents (tetrahydrofuran and Me-THF/Hexane) recycling were implemented to reduce amount of waste generated during monomer production which positively contributed to overall monomer environmental impact. 